\title{
A simple adaptive-feedback controller for identical chaos synchronization
}

\author{
Debin Huang * \\ Department of Mathematics, Shanghai University, Shanghai 200436, P.R. China
}

\begin{abstract}
Based on the invariance principle of differential equations, a simple adaptive-feedback scheme is proposed to strictly synchronize almost all chaotic systems. Unlike the usual linear feedback, the variable feedback strength is automatically adapted to completely synchronize two almost arbitrary identical chaotic systems, so this scheme is analytical, and simple to implement in practice. Moreover it is quite robust against the effect of noise. The famous Lorenz and Rössler hyperchaos systems are used as illustrative examples.
\end{abstract}

PACS number(s): 05.45.Xt, 87.17.Nn

*E-mail: dbhuang@staff.shu.edu.cn 
Since it was shown in [1] that for some chaotic systems the synchronization is possible, synchronization of (unidirectionally) coupled chaotic systems and its potential applications in engineering have been a field of great interest over a decade, see [2-4] and references cited therein. Due to the different applications, various specific synchronization schemes have been proposed in the literature, see [3] and references cited therein. However, just as what stated in [5], despite the large amount of effort many key issues remain open. One of the central questions is: Given two arbitrary identical chaotic systems, how can one design a physically available coupling scheme that is strictly guaranteed to produce stable identical synchronization motion (i.e., high-quality synchronization)? In most of the rigorous results based on the Lyapunov stability or the linear stability, the proposed scheme is very specific, but also the added controller is sometimes too big to be physically practical. One practical scheme is the linear feedback. However, in such technique it is very difficult to find the suitable feedback constant, and thus numerical calculation has to be used, e.g., the calculation of the conditional Lyapunov exponents. Due to numerical calculation, such scheme is not regular since it can be applied only to particular models. More unfortunately, it has been reported that the negativity of the conditional Lyapunov exponents is not a sufficient condition for complete chaotic synchronization, see [6]. Therefore the synchronization based on these numerical schemes can not be strict (i.e., high-qualitative), and is generally not robust against the effect of noise. Especially, in these schemes a very weak noise or a small parameter mismatch can trigger the desynchronization problem due to a sequence of bifurcations [7].

Actually, this open problem, although significant for complete chaos synchroniza- 
tion, is very difficult and cannot admit the optimization solution [3]. For example, in [5] a rigorous criteria is presented to guarantee linearly stable synchronization motion, but the criteria is so complicated that the specific numerical calculation is necessary for particular examples in practice. The similar problem was ever addressed in [8].

In this letter, we give a novel answer to the above open problem. We prove rigorously by using the invariance principle of differential equations [9] that a simple feedback coupling with the updated feedback strength, i.e., an adaptive-feedback scheme, can strictly synchronize two almost arbitrary identical chaotic systems.

Let a chaotic (drive) system be given as

$$
\dot{x}=f(x),
$$

where $x=\left(x_{1}, x_{2}, \cdots, x_{n}\right) \in \mathrm{R}^{n}, f(x)=\left(f_{1}(x), f_{2}(x), \cdots, f_{n}(x)\right): \mathrm{R}^{n} \rightarrow \mathrm{R}^{n}$ is a nonlinear vector function. And let $\Omega \subset \mathrm{R}^{n}$ be a chaotic bounded set of (1) which is globally attractive. For the vector function $f(x)$, we give a general assumption.

For any $x=\left(x_{1}, x_{2}, \cdots, x_{n}\right), x_{0}=\left(x_{1}^{0}, x_{2}^{0}, \cdots, x_{n}^{0}\right) \in \Omega$, there exists a constant $l>0$ satisfying

$$
\left|f_{i}(x)-f_{i}\left(x_{0}\right)\right| \leq l \max _{j}\left|x_{j}-x_{j}^{0}\right|, i=1,2, \cdots, n .
$$

We call the above condition as the uniform Lipschitz condition, and $l$ refers to the uniform Lipschitz constant. Note this condition is very loose, for example, the condition (2) holds as long as $\frac{\partial f_{i}}{\partial x_{j}}(i, j=1,2, \cdots, n)$ are bounded. Therefore the class 
of systems in the form of (1)-(2) include all well-known chaotic and hyperchaotic systems. Consider the variables of (1) as coupling signals, the receiver system with variables $y \in \mathrm{R}^{n}$ is given by the following equations:

$$
\dot{y}=f(y)+\epsilon(y-x)
$$

where the feedback coupling $\epsilon(y-x)=\left(\epsilon_{1} e_{1}, \epsilon_{2} e_{2}, \cdots, \epsilon_{n} e_{n}\right), e_{i}=\left(y_{i}-x_{i}\right), i=$ $1,2, \cdots, n$ denoting the synchronization error of (1) and (3). Instead of the usual linear feedback, the feedback strength $\epsilon=\left(\epsilon_{1}, \epsilon_{2}, \cdots, \epsilon_{n}\right)$ here will be duly adapted according to the following update law:

$$
\dot{\epsilon}_{i}=-\gamma_{i} e_{i}^{2}, i=1,2, \cdots, n,
$$

where $\gamma_{i}>0, i=1,2, \cdots, n$, are arbitrary constants. For the system of $2 n$ equations (which is formally called as the augment system for the convenience below), consisting of the error equation between (1) and (3), and the equation (4), we introduce the following nonnegative function

$$
V=\frac{1}{2} \sum_{i=1}^{n} e_{i}^{2}+\frac{1}{2} \sum_{i=1}^{n} \frac{1}{\gamma_{i}}\left(\epsilon_{i}+L\right)^{2}
$$

where $L$ is a constant bigger than $n l$, i.e., $L>n l$. By differentiating the function $V$ along the trajectories of the augment system, we obtain

$$
\begin{gathered}
\dot{V}=\sum_{i=1}^{n} e_{i}\left(\dot{y}_{i}-\dot{x}_{i}\right)+\sum_{i=1}^{n} \frac{1}{\gamma_{i}}\left(\epsilon_{i}+L\right) \dot{\epsilon} \\
=\sum_{i=1}^{n} e_{i}\left(f_{i}(y)-f_{i}(x)+\epsilon_{i} e_{i}\right)-\sum_{i=1}^{n}\left(\epsilon_{i}+L\right) e_{i}^{2} \leq(n l-L) \sum_{i=1}^{n} e_{i}^{2} \leq 0 .
\end{gathered}
$$

where we have assumed $x, y \in \Omega$ (without loss of the generality as $\Omega$ is globally attractive ), and used the uniform Lipschitz condition (2). It is obvious that $\dot{V}=0$ 
if and only if $e_{i}=0, i=1,2, \cdots, n$, namely the set $E=\left\{(e, \epsilon) \in \mathrm{R}^{2 n}: e=0, \epsilon=\right.$ $\left.\epsilon_{0} \in \mathrm{R}^{n}\right\}$ is the largest invariant set contained in $\dot{V}=0$ for the augment system. Then according to the well-known invariance principle of differential equations [9], starting with arbitrary initial values of the augment system, the orbit converges asymptotically to the set $E$, i.e., $y \rightarrow x$ and $\epsilon \rightarrow \epsilon_{0}$ as $t \rightarrow \infty$.

Obviously, such identical synchronization motion is strict (i.e., high-qualitative), global (as long as the chaotic attractor is globally attractive), and nonlinear stable. In particular the nonlinear global stability implies that such chaos synchronization is quite robust against the effect of noise, namely under the case of presenting a small noise the synchronization error eventually approaches zero and ultimately fluctuates around zero wheresoever the initial values start. In addition, we note that in order to reach the synchronization the variable feedback strength $\epsilon$ will be automatically adapted to a suitable strength $\epsilon_{0}$ depending on the initial values, which is significantly different from the usual linear feedback. As is well known, in the usual linear feedback scheme a fixed strength is used wheresoever the initial values start, thus the strength must be maximal, which means a kind of waste in practice. However the final strength in the present scheme depends on the initial error, thus the strength is generally equal or less than those used in the constant gain schemes. Moreover, the present control scheme does not require to determine numerically any additive parameters, and is simple to implement in practice since the technique is similar to the well-known self-adaptive controller in the control theory. Note that although the converged strength is not bigger than the linear one, theoretically the strength may be too big to be practical. However the flexibility 
of the strength in the present scheme can overcome this limitation once such case arises. For example, suppose that the feedback strength is restricted not to exceed a critical value, say $k$. In the present control procedure, once the variable strength $\epsilon$ exceeds $k$ at time $t=t_{0}$, we may choose the values of variables at this time as initial values and repeat the same control by resetting the initial strength $\epsilon(0)=0$. Namely one may achieve the synchronization within the restricted feedback strength due to the global stability of the present scheme, which is slightly similar to idea of OGY control [10]. This excellence is absent from the usual feedback scheme. In addition, in the present scheme the small converged strength may be obtained by decreasing suitably the value of parameter $\gamma$, which governs the rate of increasing of the feedback strength. As for the question on how the parameter $\gamma$ affects the convergence rate (i.e., transient time) and the final coupling strength remains to be further investigated.

Note that in the proposed scheme the coupling all the variables may be redundant to achieve synchronization, because we find from my proof that it is not necessary, e.g., one may set $\epsilon_{i} \equiv 0$ (i.e., cancelling the corresponding coupling) if $\left|e_{i}\right| \leq \mid$ $e_{j} \mid$. Actually this case exists in general due to the nonhyperbolicity of chaotic attractor, namely near a nonhyperbolic point the divergence rate of trajectories (resp. the contraction and stretching of phase space along trajectories) in some directions vanishes (or is very small), see the following examples. Although we can not give a rigorous criteria to determine only which variables can be used as the coupling signals for the general examples, in a concrete model one may determine it by the numerical technique, e.g., the calculation of the Lyapunov exponent respective 
to each direction. Of course for the low dimensional systems the optimal coupling variables may be found by directly testing again and again. Especially, if based on the calculation of the conditional Lyapunov exponents a chaotic system can be synchronized by linearly coupling a variable, then the coupling this variable in the present scheme can surely achieve synchronization, see the first example below.

Next we will give two illustrative examples. Consider the Lorenz system,

$$
\dot{x}_{1}=\beta\left(x_{2}-x_{1}\right), \quad \dot{x}_{2}=\alpha x_{1}-x_{1} x_{3}-x_{2}, \quad \dot{x}_{3}=x_{1} x_{2}-b x_{3} .
$$

The corresponding receiver system is:

$$
\dot{y}_{1}=\beta\left(y_{2}-y_{1}\right)+\epsilon_{1} e_{1}, \quad \dot{y}_{2}=\alpha y_{1}-y_{1} y_{3}-y_{2}+\epsilon_{2} e_{2}, \quad \dot{y}_{3}=y_{1} y_{2}-b y_{3}+\epsilon_{3} e_{3}
$$

with the update law (4). Now let $\beta=10, \alpha=28, b=\frac{8}{3}$. It has been well known that a suitable linear feedback in the second component may synchronize the Lorenz system, so we let $\epsilon_{1}=\epsilon_{3} \equiv 0$ (i.e., the time series of only the variable $x_{2}$ are selected as the driven signal), and set $\gamma_{2}=0.1$. The corresponding numerical results are shown in Figure 1 and Figure 2, where the initial feedback strength is set as zero. Figure 1 shows the temporal evolution of synchronization error between (7) and (8), and the variable strength $\epsilon_{2}$. Figure 2 shows that when an additive uniformly distributed random noise in the rang $[-1,1]$ (i.e., a noise of the strength 1 ) is present in the signal output $x_{2}$ of (7), the synchronization error eventually approaches zero and ultimately fluctuates slightly around zero, meanwhile the variable feedback strength is affected slightly and can not stabilize.

As the second example, we consider the Rössler hyperchaos system:

$$
\dot{x}_{1}=-x_{2}-x_{3}, \quad \dot{x}_{2}=x_{1}+0.25 x_{2}+x_{4},
$$




$$
\dot{x}_{3}=3+x_{1} x_{3}, \quad \dot{x}_{4}=-0.5 x_{3}+0.05 x_{4} .
$$

The receiver system is:

$$
\begin{gathered}
\dot{y}_{1}=-y_{2}-y_{3}+\epsilon_{1} e_{1}, \quad \dot{y}_{2}=y_{1}+0.25 y_{2}+y_{4}+\epsilon_{2} e_{2}, \\
\dot{y}_{3}=3+y_{1} y_{3}+\epsilon_{3} e_{3}, \quad \dot{y}_{4}=-0.5 y_{3}+0.05 y_{4}+\epsilon_{4} e_{4}
\end{gathered}
$$

with the update law (4). Let $\epsilon_{1} \equiv 0$ (we speculate that this is not optimal), $\gamma_{i}=$ $1, i=2,3,4$, and initial feedback strength be $(0,0,0)$, numerical results for two different cases are shown in Figure 3 and Figure 4, respectively. Figure 3 shows the hyperchaotic synchronization and Figure 4 the slight effect of a noise with the strength 0.1 which is simultaneously added to the signals $x_{2}, x_{3}$ and $x_{4}$.

The above numerical examples show that chaotic or hyperchaotic synchronization can be quickly achieved by the present controller (i.e., the transient time to synchronization is very short). In addition, we find from these examples that such synchronization is robust against the effect of noise, namely if the expected synchronization means that the synchronization error is eventually smaller than a threshold value (not tends to zero), then the present scheme is physically feasible in the noisy case. Moreover, by comparing the converged feedback strength and the corresponding feedback signals we find that the coupling is indeed small in the examples. In addition by testing the other chaotic systems including the Rössler system, Chua's circuit, and the Sprott's collection of the simplest chaotic flows we find that the coupling only one variable is sufficient to achieve identical synchronization of a three-dimensional system.

In conclusion, we have given a novel answer to the open problem in the field 
of identical chaos synchronization. In comparison with the previous methods, the proposed scheme supplies a simple, analytical, and (systematic) uniform controller to synchronize strictly two arbitrary identical chaotic systems satisfying a very loose condition. The technique is simple to implement in practice, and quite robust against the effect of noise. The control idea may be also generalized to the case of the discrete chaotic systems. We also believe that such simple synchronization controller will be very beneficial for the applications of chaos synchronization. Especially the similar control scheme has been successfully used to stabilize the chaotic neuron model [11], and hence the proposed adaptive-feedback synchronization controller can be used to explore more reasonably the interesting dynamics found in neurobiological systems, i.e., the onset of regular bursts in a group of irregularly bursting neurons with different individual properties [12].

Acknowledgments: The author is in debt to all referees including those consulted by PRL and PRE for their valuable suggestions. This work is supported by the National Natural Science Foundation of China (10201020; 10432010).

\section{References}

[1] L.M. Pecora and T.L. Carroll, Phys. Rev. Lett. 64, 821 (1990); Phys. Rev. A 44, 2374 (1991).

[2] K.M. Cuomo and A.V. Oppenheim, Phys. Rev. Lett. 71, 65 (1993); L. Kocarev and U. Parlitz, Phys. Rev. Lett. 74, 5028 (1995); U. Parlitz, Phys. Rev. Lett. 76, 1232 (1996); Chaos 7, (4) (1997); Chaos 13, (1) (2003). 
[3] S. Boccaletti, et al, Phys. Reports 366, 1 (2002).

[4] Debin Huang and Rongwei Guo, Chaos 14, 152 (2004); Debin Huang, Phys. Rev. E 69, 067201 (2004).

[5] R. Brown and N.F. Rulkov, Phys. Rev. Lett. 78, 4189 (1997); Chaos 7, 395 (1997).

[6] J.W. Shuai, K.W. Wong, and L.M. Cheng, Phys. Rev. E 56, 2272 (1997); C. Zhou and C.H. Lai, Phys. Rev. E 58, 188 (1998); Physica D 135, 1 (2000).

[7] P. Ashwin, J. Buescu and I. Stewart, Phys. Lett. A 193, 126 (1994); S.C. Venkataramani, B.R. Hunt and E. Ott, Phys. Rev. Lett. 77 (1996) 5361; Phys. Rev. E 54 (1996) 1346; E. Barreto, P. So, B.J. Gluckmann and S.J. Schiff, Phys. Rev. Lett. 84 (2000) 1689.

[8] D.J. Gauthier and J.C. Bienfang, Phys. Rev. Lett. 77, 1751 (1996); I. Grosu, Phys. Rev. E 56, 3709 (1997); K. Josic, Phys. Rev. Lett. 80, 3053 (1998); O. Morgul, Phys. Rev. Lett. 82, 77 (1999).

[9] J.P. Lasalle, Pro. Natl. Acad. Sci. 46, 363 (1960); IRE Trans. Circuit Theory 7, $520(1960)$.

[10] E. Ott, C. Grebogi and J.A. Yorke, Phys. Rev. Lett. 64, 1196 (1990).

[11] Debin Huang, Phys. Rev. Lett. 93, 214101 (2004).

[12] N.F. Rulkov, Phys. Rev. Lett. 86, 183 (2001); M.G. Rosenblum and A.S. Pikovsky, Phys. Rev. Lett. 92, 114102 (2004). 

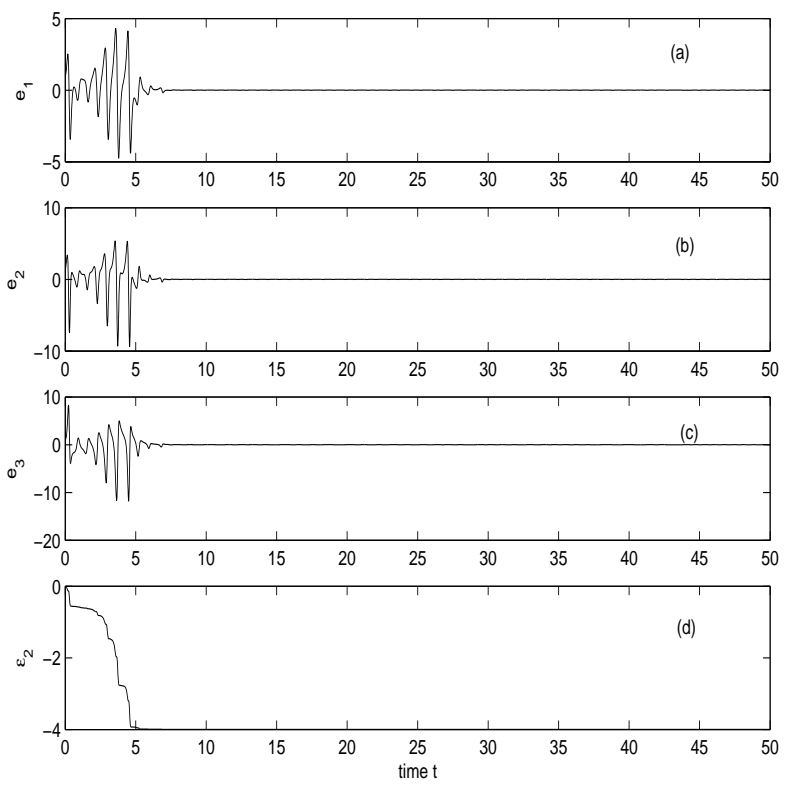

FIG.1. The chaos synchronization between (7) and

(8) is achieved by only the signal $x_{2}$, where (a)-(c)

show temporal evolution of the synchronization error and (d) shows the evolution of the corresponding feedback strength $\epsilon_{2}$. Here the initial values of $(x, y, \epsilon)$ is set as $(2,3,7,3,4,8,0)$. 

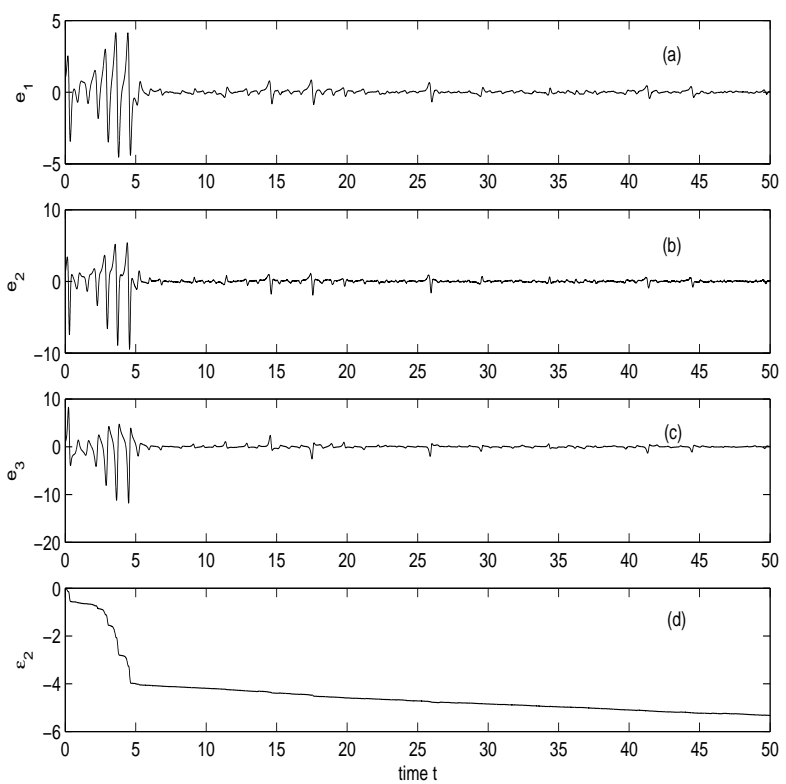

FIG.2. (a)-(d) show the effect on the synchroniza-

tion error and the feedback strength in Figure 1 when a noise of the strength 1 is present in the signal output $x_{2}$ of (7). 


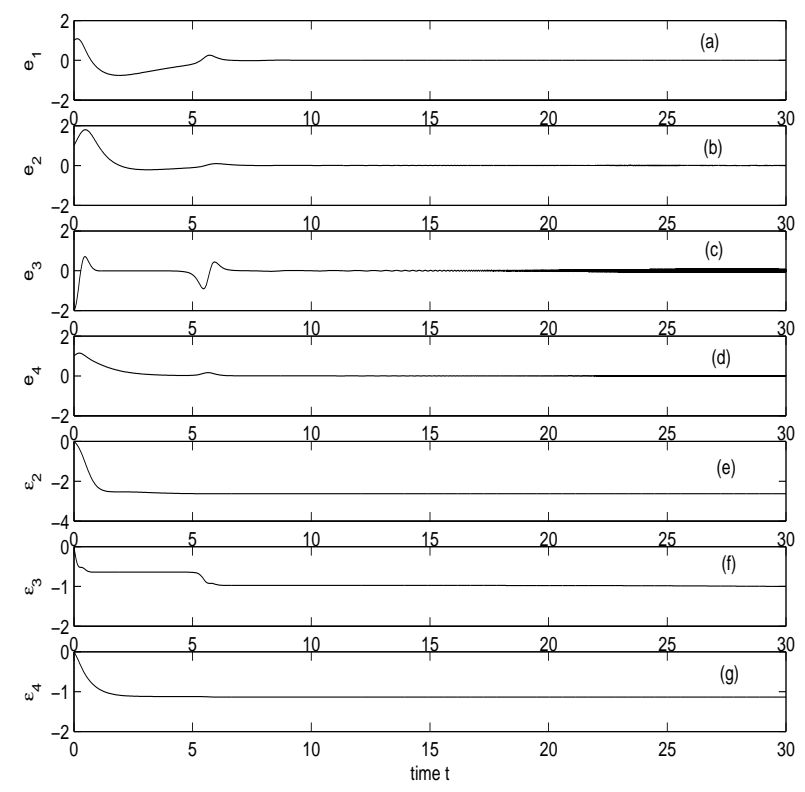

FIG.3. (a)-(g) show the hyperchaotic synchronization between systems (9) and (10), and temporal evolution of the corresponding feedback strength, where only three variables of $(9), x_{i}, i=2,3,4$ are selected as the driven signals. Here the initial values of $(x, y, \epsilon)$ is set as $(2,3,7,10,3,4,5,11,0,0,0)$ 


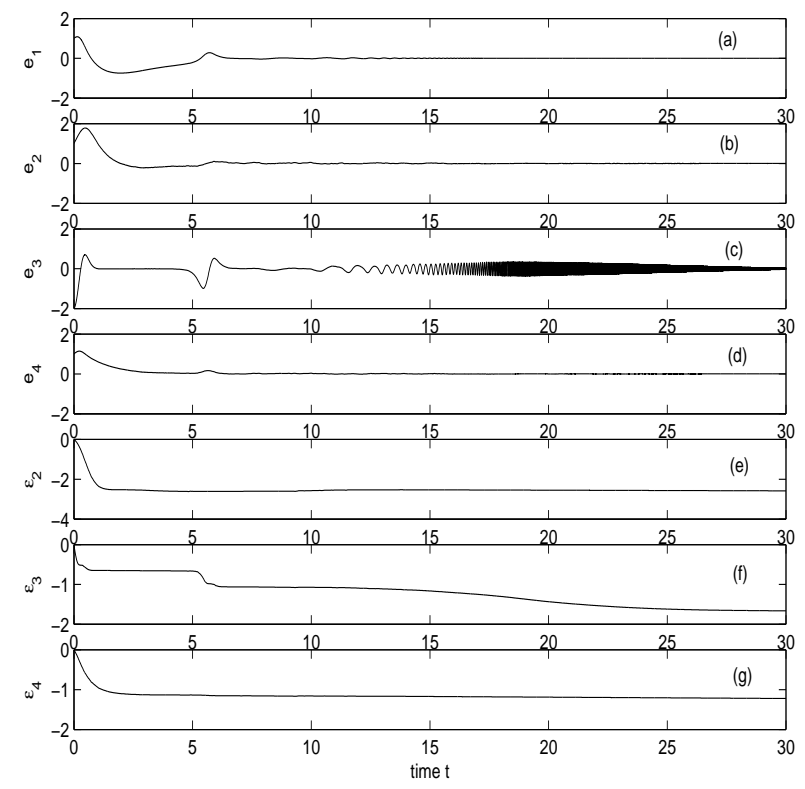

FIG.4. (a)-(g) show the effect on the synchroniza-

tion error and the feedback strength in Figure 3 when

a noise of the strength 0.1 is simultaneously added to the signal outputs $x_{2}, x_{3}$ and $x_{4}$ of (9). 